need to mitigate, respond to, and recover from a health emergency.

Objectives: To give leadership, clarify roles and responsibilities, facilitate and coordinate planning, achieve an effective and consistent response, and improve awareness and capability.

Methods: The health sector is unique in that it deals daily with large and small-scale emergencies from individual tragedies to community-wide events. Hospital front doors are always open. Coupled with an unrelenting pressure on resources and increasing public expectations, this means that many parts of most health sectors have well-advanced plans for dealing with surges in demand. Workforce planning, quality control, immunization strategies, and hospital emergency plans are all aspects of existing work that the National Health Emergency Plan gathered.

As a first step in the process sector and Ministry projects were reviewed to identify those aligned with these objectives. Gaps were identified with help from consultative groups. Projects were begun which filled in these gaps and pulled the disparate strands of work together into cohesive work streams. Responsibilities were decided. A program of meetings, monitoring, and reports was established.

Results: The NHEP has largely taken shape and in the process has helped highlight further capacity and structural issues that need to be resolved to help achieve a higher level of preparedness. It will continue to evolve and develop and a lot more work is yet to come.

Conclusions: The New Zealand health sector is better prepared now than it was for SARS or the Avian flu and, fortunately, is still improving.

Keywords: health; National Health Emergency Plan (NHEP); New

Zealand; preparedness; severe acute respiratory syndrome (SARS) Prebosp Disast Med 2005;20(2):s48-s49

\section{The State of Connecticut Emergency Credentialing System}

J. Arnold; C. Cannon; E. Forte; J. Paturas

Yale-New Haven Center for Emergency Preparedness,

New Haven, Connecticut, USA

Introduction: Disasters may produce a maldistribution of volunteer healthcare workers to acute care hospitals, sometimes causing uncertainty regarding their qualifications to provide medical care and other services. The development and implementation of a regional emergency credentialing system (ECS) for volunteer healthcare workers in the state of Connecticut are described.

Methods: A statewide coalition of key stakeholders in the ECS, including the State of Connecticut Department of Public Health, the Connecticut Hospital Association, and the Yale-New Haven Health System (YNHHS), was convened in November 2002. The coalition developed a plan to: (1) engage all 32 acute care hospitals in Connecticut in the ECS through written agreements that ensure that participating hospitals will recognize the credentials of volunteer healthcare workers credentialed at other participating hospitals; and (2) recruit licensed physicians and mid-level practitioners to serve as volunteer medical providers for those hospitals. Concomitantly, state legislation was adopt- ed, which incorporated these volunteer healthcare workers into the Connecticut Medical Reserve Corps, providing them with liability insurance and disability coverage. The YNHHS was selected to manage the ECS and maintain its electronic database. In an actual event, acute care hospitals would contact the ECS to request the needed numbers and types of pre-credentialed healthcare workers $(\mathrm{HCWs})$. Volunteer healthcare workers will be contacted by the ECS and then dispatched to the requesting hospital. In 2005, the ECS will begin recruiting other types of volunteer $\mathrm{HCWs}$, including nurses, physical therapists, laboratorians, and radiographers.

Results: Acute care hospitals began participating in the ECS in December 2003. Thirty-one of 32 (97\%) acute care hospitals in Connecticut have agreed to participate in the ECS, and recognize the credentials of participating volunteer healthcare workers at their institutions. Physicians and mid-level practitioners began participating in the ECS in January 2004. As of November 2004, 1,586 (9\%) of 18,350 licensed physicians and mid-level practitioners in Connecticut have agreed to serve as volunteer medical providers. Participating physicians represent the range of medical specialties. The Joint Commission on the Accreditation of Healthcare Organizations has designated the State of Connecticut ECS as a national model for the United States. The ECS has yet to be activated during an actual event.

Conclusion: Successful implementation of an ECS for acute care hospitals depends on the participation of key stakeholders during development, the sequential roll-out to participating hospitals and volunteer healthcare workers, and overcoming key barriers, such as the lack of liability insurance for volunteer healthcare workers.

Keywords: Connecticut; emergency credentialing system; healthcare workers ( $\mathrm{HCWs}$ ); volunteer

Prehosp Disast Med 2005;20(2):s49

\section{How Can We Reduce On-Scene Time in an Urban Region? \\ A. Djalali}

NMRI (Pasteur Institute of Iran), Iran

Tehran, the capital of Iran, has a population of 10 million. It is subject to crowding and traffic jams. In 1998, there were 20 prehospital emergency medical services (EMS) stations, staffed by intermediate emergency medical technicians (EMTs). At that time, the average time between a patient's call and the EMS arrival on-scene was almost 30 minutes.

An assessment to find the causes of these long delays was conducted. The results showed that some of the main causes were: (1) traffic jams, especially in the city center and squares; (2) not enough ambulances; (3) not enough EMS stations; (4) delays in EMS team starting its duties; (5) an overload of false duties; (6) an overload of incorrect diagnoses; and (7) unnecessary transport of patients to hospital.

The prehospital EMS management made the following changes over the next few years: (1) increasing the number of ambulances; (2) cooperating with the firefighting system to increase the number of stations; (3) additional edu- 
cation of EMTs; (4) moving the ambulance parking inside EMS stations; (5) using standby ambulances and EMS teams in crowded squares; (6) establishing an air ambulance (helicopter) in Tehran for rapid medical responses to highway scenes; (7) creating motorcycle teams for rapid medical response in crowded parts of the city; (8) setting up professional teams of nurses and general practitioners; and (9) having general practitioners inside dispatch room of EMS centers advise EMS teams.

By 2002, on-scene time for EMS teams from Tehran 115 center was almost 10-15 minutes (median $=10$; mean $=15$ ). This time was eight and five minutes for the standby ambulance and motorcycle routes, respectively. Also, the air ambulance had an on-scene time below eight minutes. Although there are still some problems and delays with EMS team duties, the critical on-scene time has dropped closer to the "golden time" of five to eight minutes.

Keywords: emergency medical services (EMS); Iran; on-scene time; traffic

Prehosp Disast Med 2005;20(2):s49-s50

\section{Prospective, Cross-Sectional Analysis of Hospital Bed} Surge Capacity in Selected San Diego Hospitals I. Jacoby; ${ }_{i}^{1-4}$ D. Davis ${ }^{1,2}$ J. Poste; ${ }^{5}$ T. Hicks; ${ }^{6}$ D. Polk; T. Rymer ${ }^{2-4}$

1. University of California at San Diego (UCSD) Department of Emergency Medicine, San Diego, California USA

2. UCSD School of Medicine, La Jolla, California USA

3. San Diego Disaster Medical Assistance Team (DMAT), San Diego, California USA

4. UCSD Office of Emergency Preparedness and Response, San Diego, California USA

5. John Muir College, University of California, San Diego, California USA

6. Sharp Healthcare, San Diego, California USA

7. Scripps Memorial Hospital, La Jolla, California USA

Introduction: Traditional strategies to determine hospital surge capacity have relied on cross-sectional, hospital census data. This likely underestimates the true surge capacity in the event of a mass-casualty incident.

Objective: To more accurately determine hospital bed surge capacity using physician and nurse manager assessments at multiple facilities.

Methods: Overnight and day shift nurse managers from each inpatient unit at four different hospitals were approached to make assessments on all patients under their care. Physicians at two academic institutions also were approached for comparison. Age, gender, ward type, and admission diagnosis were recorded. In addition, assessments were made for each patient as to their disposition at two, 24, and 72 hours following a mass-casualty incident. Transfers to a lower level of care, including a hypothetical "on-site nursing facility" or discharge to home were included. A physical assessment of each hospital also was made to determine whether enough space was available for patients transferred to the "on-site nursing facility." Data were reported descriptively, and comparisons were made between overnight and day shift nurses and between physician and nurse manager assessments.
Results: A total of 1,745 assessments on 776 patients by 82 nurse managers and 25 physicians from the four institutions were included. Nurse managers assessed approximately one-third of all patients as dischargeable at 24 hours and approximately one-half at 72 hours; one-quarter of patients were assessed as being transferable to an "on-site nursing facility" at both time points. Physicians were more likely than nurse managers to send patients to such a facility or discharge them, but less likely to transfer patients out of the intensive care unit.

Conclusions: A large proportion of inpatients can be discharged within 24 and 72 hours. Additional beds can be made available if an "on-site nursing facility" is made available. Both physicians and nurse managers should be included on the team that makes patient dispositions in the event of a mass-casualty incident.

Keywords: assessments; California; hospital beds; inpatients; masscasualty incident; nurse managers; on-site nursing facility; physicians; San Diego; surge capacity; transfer

Prehosp Disast Med 2005;20(2):s50

Sensitivity and Specificity of Advanced Medical Priority Dispatch System (AMPDS) in Detecting Cardiac Arrests in Melbourne

J. Flynn, ${ }^{1}$ F. Archer; ${ }^{1}$ I. Dale ${ }^{2}$

1. Monash University, Victoria, Australia

2. Metropolitan Ambulance Service (Melbourne), Victoria, Australia

Introduction: To ensure that patients suffering from an out-of-hospital cardiac arrest receive maximal ambulance response, it must be identified as cardiac arrest by the emergency call taker. In Melbourne, the Metropolitan Ambulance Service uses the Advanced Medical Priority Dispatch System (AMPDS) to aid call taking.

Objective: This study aims to determine the sensitivity and specificity of the AMPDS in detecting cardiac arrest emergency calls in Melbourne and to analyze possible areas for improvement.

Method: Dispatch records for suspected cardiac arrests (priority zero) from January 2001 through March 2003 were compared with the Victorian Ambulance Cardiac Arrest Registry (VACAR). This identified those cardiac arrests that were identified correctly by AMPDS, the cardiac arrests not identified, and priority zero cases, which were not cardiac arrests. Where the dispatch code was inaccurate, further analysis was undertaken to identify possible areas for improvement.

Results: The sensitivity of the AMPDS in detecting cardiac arrest was $76.7 \%$ (95\% Confidence Interval (CI) 73.6-79.8\%), specificity was $99.2 \%$ (95\% CI 99.1-99.3\%). Cardiac arrests given lower priorities were allocated 58 different dispatch codes. No method for better identification of cardiac arrest was evident in the limited data available. Of the cases that were dispatched as priority zero, but which were not cardiac arrests, $37.2 \%$ had a Glasgow Coma Scale Score of 13 and normal or near normal ventilatory rate. Identifying this during emergency calls may reduce the dispatch of unnecessary maximal responses, reducing road hazards and allowing the more efficient resource use. 